- Next-Generation Suborbital Researchers Conference in Broomfield, Colorado, on 18-20 December.

Among the ballooning companies that accept scientific payloads are Raven Aerostar of Sioux Falls, South Dakota, and Near Space Corporation of Tillamook, Oregon. World View has made a splash in the past year by developing a standardized 'Stratollite' platform that dangles beneath its balloons. A payload that might cost more than US\$1 million to fly on a NASA balloon could fly for tens of thousands of dollars on World View if it shares a Stratollite with other experiments, Stern says.

In 2018, World View aims to fly up to four times a month, says Jane Poynter, the company's chief executive. Each balloon would lift one Stratollite carrying one or more experiments into the stratosphere. The longest flight so far lasted just over five days, but Poynter says the company hopes to conduct weekslong flights in the near future.

Robert Grimm, a planetary scientist at the Southwest Research Institute, flew an experiment on a World View balloon in October to test designs for a possible mission to Venus. The planet's surface is too hot for equipment to survive for long, but conditions high in Venus's atmosphere are much more temperate - meaning that scientists could use balloons as a way to study the planet for months, rather than minutes or hours.

After taking off in Idaho, the balloon soared for 500 kilometres before touching down in Montana. Like a high-flying metal detector, Grimm's on-board equipment measured changes in electrical properties within a granite-rich mountain range below. Collecting such data over Venus could illuminate the geology at or beneath the planet's surface, says Grimm, who hopes to fly further experiments in May.

World View has also developed ways to hold its balloons nearly stationary over a point of interest. The company directs the balloon up

\section{"We need} observations from balloons, because they'rejust so powerful." and down to catch the wind and keep the craft in approximately the same location. Google's parent, Alphabet, uses a similar approach to keep its Project Loon balloons in one spot. The company has been testing whether the balloons, built by Raven Aerostar, can provide Internet connectivity in places such as Puerto Rico, following last September's devastating Hurricane Maria.

NASA is developing advanced balloon technology for scientists, including its 'superpressure' balloons that can fly for up to 100 days - a period suitable for long-term studies such as certain astronomical observations. But the work is expensive and technologically challenging. For many experiments, "the World
View flights are actually there", says Thomas Zurbuchen, NASA's associate administrator for science in Washington DC. "We're really interested in doing some science on these new platforms." (The agency funded Grimm's World View flight.)

To Adrienne Dove, a planetary scientist at the University of Central Florida in Orlando, stratospheric balloons offer a new opportunity to explore the physics behind spaceflight. She studies how dust clumps together in lowgravity conditions - important for lunar and planetary exploration - and has worked with sounding rockets and the 'vomit comet' aeroplanes that create low gravity for short periods during their parabolic flights. "My interest is in developing microgravity capability on balloons, which currently doesn't exist," she says.

Looking even further into the future is Siddharth Krishnamoorthy, an aerospace engineer at the Jet Propulsion Laboratory in Pasadena, California. His team wants to use stratospheric balloons to listen for low-frequency infrasound signals coming from earthquakes, as a test for possible future missions to probe for seismic activity on Venus.

That would mean floating in the stratosphere above earthquake-prone places such as Oklahoma or California, listening for infrasound signals and pretending they are on Venus. "Yes, it's cool," says Krishnamoorthy. -

Slow-starter seeks to catch up with rival techniques.

\section{BY DAVIDE CASTELVECCHI}

$\mathrm{I}$ $\mathrm{n}$ the next few weeks, a research group at the Delft University of Technology in the Netherlands expects to receive an important package. Its contents promise to increase competition in the race to produce useful quantum computers.

Shipped from the research-and-development facilities of semiconductor giant Intel in Hillsboro, Oregon, the parcel holds the first quantum computer manufactured with the techniques used to fabricate silicon chips in conventional computers. Although the silicon method currently lags behind other approaches to building quantum computers, the company hopes that the technique could accelerate the development of devices that go beyond proof-of-concept curiosities, says James Clarke, who heads Intel's quantum-hardware development. "I think you'll hear a lot about silicon quantum computing this year," Clarke says.

The relatively modest device represents the latest move in the push to give silicon a boost over other approaches. Some scientists also see promise in the silicon route. Physicists such as Michelle Simmons at the University of New South Wales (UNSW) in Sydney, Australia, are developing their own ways of building quantum computers using silicon. In May 2017, she founded an Aus $\$ 83$ million (US\$65 million) start-up called Silicon Quantum Computing, backed in part by the Australian government.

Quantum computers aim to exploit two small-scale phenomena to outperform their classical counterparts, which encode bits of information as 0 s and $1 \mathrm{~s}$. In the quantum world, units of information are called qubits, and each qubit can exist simultaneously in a 'superposition' of both 0 and 1 . Two bits can also be entangled, so that the state of one qubit determines the state of its partner. This enables quantum devices to conduct calculations in parallel.

Physicists in many labs have developed prototype quantum computers, which often operate at temperatures close to absolute zero. The frontrunners in the race use one of two methods to encode the qubits: single ions held in traps, or oscillating currents in superconducting loops. Both systems require exquisite control: the ion technique uses complex laser systems to read and write each qubit, and superconducting qubits must each have a device to control them using radio waves. 


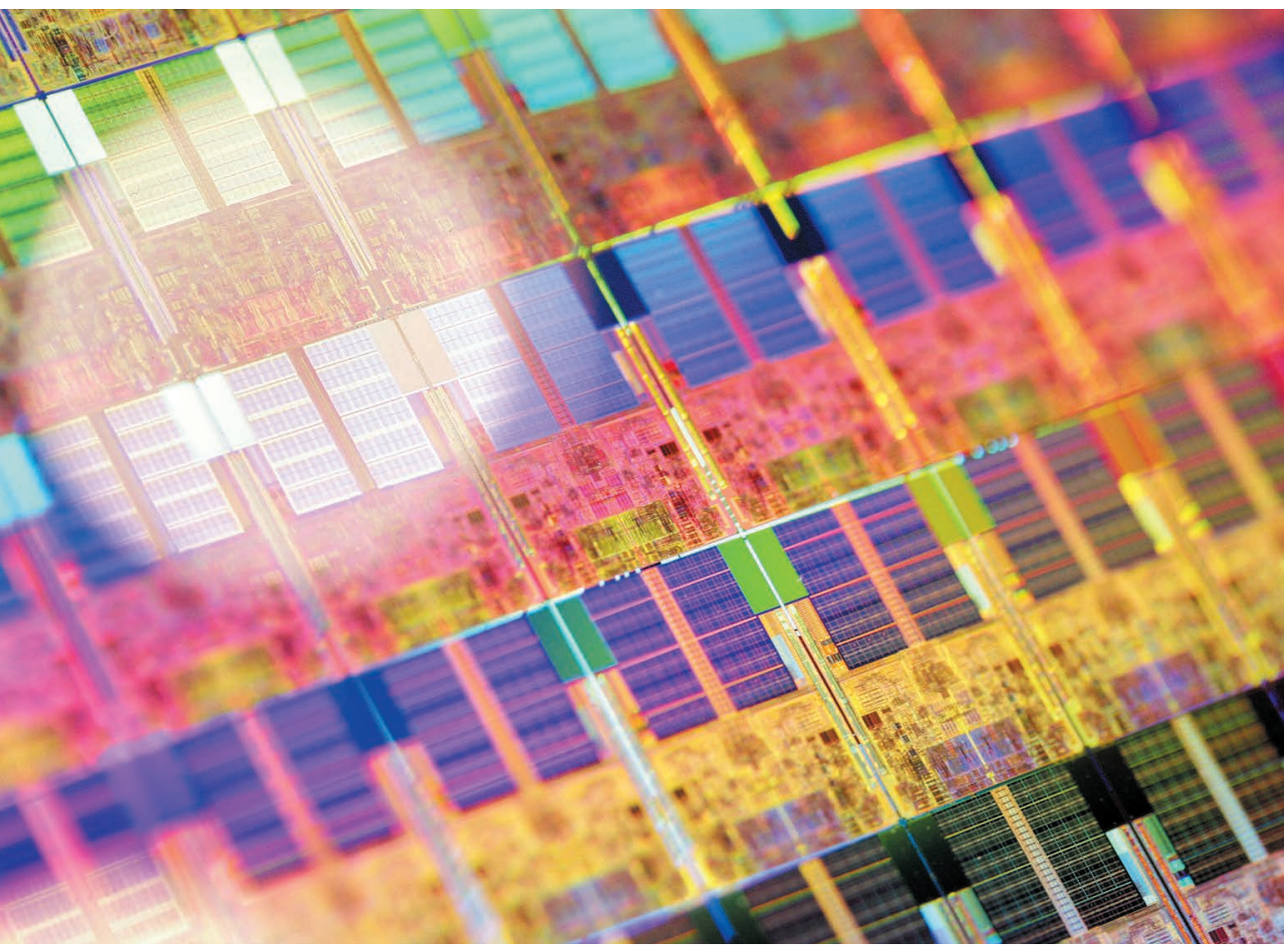

Techniques for fabricating conventional silicon chips could be used to make quantum devices.

Proponents of the silicon technique see major advantages in using a semiconductor to code qubits. They can be manipulated much more simply using microscopic electric leads etched right onto the chip. And if the same large-scale manufacturing techniques for making chips could be transferred to the quantum realm, it could become easier to turn the technology into commercial products.

\section{A LONG ROAD}

The idea of building quantum computers out of silicon is not new. Bruce Kane, an experimental physicist now at the University of Maryland in College Park, first suggested encoding qubits in the magnetic orientation, or 'spin', of phosphorus nuclei embedded in silicon 20 years ago ${ }^{1}$. At about the same time, David DiVincenzo, a theoretical physicist then at IBM in Yorktown Heights, New York, and his collaborator Daniel Loss at the University of Basel in Switzerland proposed a way of storing information in the spins of mobile electrons inside semiconductors ${ }^{2}$. Both proposals led to a number of experimental demonstrations but, for a long time, the quality of the materials limited progress.

Building a quantum computer using silicon took years of "not very flashy" developments in materials science and engineering, says physicist Jason Petta of Princeton University in New Jersey. Physicists at the UNSW Centre for Quantum Computation and Communication Technology, which Simmons directs, have done much of that groundwork. And Simmons developed a manufacturing technique that requires fewer control leads, preventing inevitable issues of crowding once quantum

devices scale up, she says. "I want to engineer everything out that isn't essential and make things as simple as possible."

In 2017, two groups reached a milestone when they designed the first fully controllable two-qubit devices in silicon. Petta and his
"I want to

engineer everything out that isn't essential and make things as simple as possible."

turing multiple-qubit electron-spin devices for Vandersypen, in the same type of factory where it develops microprocessor-fabrication techniques. Industrial partners can help by providing reliably identical devices, he says.

"We hope that we can accelerate spin qubits to compete" with the more mature approaches, Clarke says. Simmons' start-up aims to build a ten-qubit machine within five years. Google, IBM and a number of other companies and academic labs are all using different techniques to build quantum computers with around 50 superconducting qubits - and so is Intel itself, which is hedging its bets by supporting more than one technical approach.

1. Kane, B. E. Nature 393, 133-137 (1998).

2. Loss, D. \& DiVincenzo, D. P. Phys. Rev. A 57, 120-126 (1998).

3. Zajac, D. M. et al. Science http://dx.doi. org/10.1126/science.aao5965 (2017).

4. Watson, T. F. et al. Preprint at https://arxiv.org/ abs/1708.04214 (2017).

\section{PUBLISHING}

\section{Elsevier grants a reprieve}

\section{It allows German institutions continued journalaccess.}

\section{BY QUIRIN SCHIERMEIER}

$\mathrm{T}$ The Dutch publishing giant Elsevier has granted uninterrupted access to its paywalled journals for researchers at around 200 German universities and research institutes that had refused to renew their individual subscriptions at the end of 2017.

The institutions had formed a consortium to negotiate a nationwide licence with the publisher. They sought a collective deal that would give most scientists in Germany full online access to about 2,500 journals at roughly half the price that individual libraries have paid in the past. But talks broke down and, by the end of 2017, no deal had been agreed. Elsevier now says that it will allow the country's scientists to access its paywalled journals without a contract until either a national agreement is reached or 200 individual contracts are hammered out.

The two sides had "constructive conversations well into December", says Harald Boersma, a spokesman for Elsevier. "We will continue our conversations in the first quarter of 2018 to find an access solution for German researchers in 2018 and a longer-term national agreement," he says. "Where access agreements ended, we have informed these institutions that we would maintain access to our content while we continue to work with the German Rectors' Conference [which leads negotiations for the consortium] on a solution and specifically a one-year extension to existing contracts covering 2018."

Günter Ziegler, a mathematician at the Free University of Berlin and a member of the consortium's negotiating team, says that German researchers have the upper hand in the talks. "Most papers are now freely available somewhere on the Internet, or else you might choose to work with preprint versions," he says. "Clearly our negotiating position is strong."

Academic-publishing experts around the world are keenly observing the situation in Germany. The nationwide deal sought by scientists includes a open-access option, under which all corresponding authors affiliated with German institutions would be allowed to make their papers free to read and share for anyone in the world. This would be a milestone for global efforts to make the results of publicly funded research immediately and freely available to scientists and the wider public, they say. 